ISSN: 2600-5859

\title{
La utilización de la tecnología en el desarrollo del lenguaje de los niños de 4 años () (1) @()
}

\section{The use of technology in the language development of 4-year old}

Paola de los Ángeles Bonilla Morales. ${ }^{1}$, Elsa Verónica Troya Ortiz. ${ }^{2}$ \& Víctor Peñafiel Gaibor. ${ }^{3}$ Recibido:05-01-2020 / Revisado:22-01-2020 /Aceptado: 03-02-2020/ Publicado: 05-03-2020

\begin{abstract}
DOI: https://doi.org/10.33262/concienciadigital.v3i1.2.1174

The comparative research establishes the connection between technology use and language development in 4-year-old children. Furthermore, this research included 34 kids who attend to Preschool at Sagrada Familia School. The 34 kids were assigned into two groups, an experimental group and a control group. Qualitative and quantitative methods were used throughout the information gathering techniques. So, to get the kids' language development level, language subtest from psychomotor development test TEPSI was used. The test's images were used with the experimental group while technology was used with the control group. The test results were obtained, and they were analyzed by a statistics program. Additionally, the techno-logy use promoted the language assessment obtaining appropriate scores in relation to the kids' age. Definitely, technology has to be taken as a supportive key to language development on digital natives. It is in that way how new research areas emerge about technology and its therapeutic use in early stimulation areas.
\end{abstract}

Keywords: Technology, Language, Digital natives, TEPSI

\section{Resumen}

El estudio comparativo establece la relación entre la utilización de la tecnología y el desarrollo del lenguaje de los infantes en el cuarto año de vida. En el presente estudió se trabajó con una población de 34 niños que asisten al Inicial 2 de la Unidad Educativa Sagrada Familia que fueron divididos en dos grupos: un normal y otro de control. Los métodos utilizados fueron cualitativos

\footnotetext{
${ }^{1}$ Universidad Técnica de Ambato, Facultad de Ciencias de la Salud, Ecuador, pbonilla2932@uta.edu.ec

2Universidad Técnica de Ambato, Facultad de Ciencias de la Salud, Ecuador, elsavtroya@uta.edu.ec

${ }^{3}$ Universidad Técnica de Ambato, Facultad de Ciencias de la Salud, Ecuador, vi.penafiel@uta.edu.ec
} 
ISSN: 2600-5859

y cuantitativos a través de las técnicas de recolección de la información. Así, para obtener el nivel de desarrollo del lenguaje de los niños, se empleó el sub test de lenguaje del test de desarrollo psicomotor TEPSI. Al grupo normal se aplicó las láminas del test, mientras que, al grupo de control se trabajó empleando el mismo test, pero aplicando la tecnología. Seguidamente, se obtuvieron los resultados del test utilizado en los dos grupos y se procesó la información mediante el uso de un estadístico. Por otra parte, el análisis probó que la utilización de la tecnología favorece la valoración del lenguaje, consiguiendo puntajes adecuados para la edad del niño. En definitiva, se evidencia que la tecnología debe ser vista como una herramienta de apoyo para el desarrollo del aprendizaje del lenguaje en niños de 4 años. De esta forma, vemos que se abre nuevas líneas de investigación en cuanto se refiere a la implementación de la tecnología como uso terapéutico en áreas de la estimulación temprana.

Palabras claves: Tecnología, Lenguaje, Nativos Digitales, TEPSI

\section{Introducción}

A nivel mundial la tecnología ha permitido mejorar el bienestar humano y se ha impregnado rápidamente en la sociedad, además, se constituye como un servicio básico que ha mejorado el estilo de vida de las personas. Analizando la obre de V. Gordon Childe (1997) que hace referencia a dos grandes revoluciones históricas. Vemos que, en primera instancia, el ser humano se transforma de cazador-recolector a una vida sedentaria, que se debió a la fabricación de los alimentos. Mientras que, en la segunda el ser humano cambia de la prehistoria a la historia escrita, debido al desarrollo de las primeras formas de escritura y de esta manera se formó la base de la civilización. Además, el autor propone un criterio cuantitativo, el cual explica que el desarrollo progresivo es sinónimo de revolución tecnológica. Así como sucedió en el periodo de transición del paleolítico al neolítico, el cual mostró un paso de progreso y gracias al desarrollo de la producción de alimentos, la población incrementó diez veces más al contrario de los grupos nómadas.

En este sentido, haciendo una analogía, de acuerdo con el Fondo de las Naciones Unidas para la Infancia UNICEF, las innovaciones tecnológicas han acelerado el ritmo de vida, mientras que, para la Organización Mundial de la Salud, la tecnología debe implementarse de manera sostenible y equitativa. También, la Organización de Cooperación y Desarrollo Económicos de los países más ricos del mundo manifiesta que "la experiencia en materia de tecnologías de la 
ISSN: 2600-5859

información y la comunicación (TIC) ha tenido un gran impacto sobre la participación en la fuerza laboral y los salarios en países como Australia y los Estados Unidos. Los adultos sin experiencia en TIC, incluso cuando están empleados, probablemente ganen menos que aquellos con conocimientos de TIC. Otros estudios de poblaciones adultas en países como la India y Túnez reflejan conclusiones similares" (UNICEF, 2017). Así, vemos que los países en desarrollo muestran adelantos en campos como la industria, la economía, la educación, la cultura, entre otras.

No obstante, América Latina ha ensayado múltiples mecanismos que ha permitido dejar atrás una sociedad de desconocimiento científico.

Sin embargo, debido al auge de las tecnologías y los cambios socioculturales de los últimos años, el ambiente adecuado para el desarrollo del lenguaje ha sido suplantado por aparatos tecnológicos diseñados para que cualquier persona los use. Por esta razón es necesario explicar si las tecnologías son favorables en la adquisición de habilidades, tomando en cuenta la edad, el uso correcto y la aplicación de un horario.

En esta dirección, la Organización Mundial de la Salud, emitió una guía basada en 277 artículos y 10 estudios, en cinco países con casi 7500 participantes. Esta menciona que inferior a los dos años no se debe ver televisión, ni jugar con pantallas, mientras que, los niños entre dos y cincos años pueden utilizar dispositivos tecnológicos como mínimo una hora al día. De acuerdo con estas recomendaciones a nivel mundial, se busca prevenir problemas de salud en niños, niñas y adolescentes. Así, 40 millones de niños menores de cinco años (un 5,9\% del total) en el mundo tienen sobrepeso.

En contraste con ello, vemos que la tecnología es un conjunto de conocimientos de base científica que permite describir, explicar, diseñar y aplicar soluciones técnicas a problemas prácticos de forma sistemática y racional (Quintanilla, 1998). Dado que el mundo actual está inundado de tecnología, al hacer una llamada, encender un Smart TV o navegar en la web, absolutamente, todo es tecnología. Esta ha ayudado en la solución de problemas de una manera ordenada y satisfactoria, puesto que, la ayuda de las TIC ha tenido un papel fundamental en múltiples campos de la vida actual.

En la actualidad, la tecnología constituye un servicio básico que ha mejorado el estilo de vida de las personas a nivel mundial. Así, en Ecuador se registra un incremento del 12,1\% en cuanto se refiere a dispositivos portátiles, tablet y laptop. Es decir, 9 de cada 10 hogares utiliza 
ISSN: 2600-5859

tecnología. En este sentido, con el auge de las tecnologías y los cambios socioculturales de los últimos años, el ambiente adecuado para el desarrollo del lenguaje ha sido suplantado por aparatos tecnológicos.

De la misma forma, la tecnología constituye aparatos modernos formados por piezas y aplicaciones portátiles especializadas para cada utilización.

En el año 2007, para algunos autores, se consideraba que la tecnología era una buena práctica; de esta manera se denominó "Las TIC para la igualdad. Nuevas tecnologías y atención a la diversidad" (Cabero, Córdoba \& Fernández, 2010). Asimismo, la tecnología ha sido vista como una herramienta terapéutica y educativa. De este modo, las TIC han repercutido cada vez más en los espacios domésticos y han influido en el estudio de aplicaciones o modificaciones de estos, necesarios para lograr adaptar a distintos grupos de personas. "Se ha elevado la diversidad de funciones para las cuales son utilizadas, y se ha incrementado las tipologías de TIC que se encuentran presentes" (Córdova, Fernández y Cabero, 2012).

Considerando que el sistema nervioso, específicamente el neocórtex se involucra en el lenguaje verbal y no verbal, esto ha llevado a definir al lenguaje como un proceso ligado al funcionamiento de neuronas (Alfonso y López, 2013). Podemos acotar la importancia de brindar al niño un estímulo no solo auditivo, sino que sea visual ya que los estímulos auditivos, inicialmente son procesados en el órgano de Corti situado en el oído interno.

Desde este lugar se transmite información a través del nervio coclear, dirigiéndose a los tubérculos cuadrigéminos posteriores, localizados en el tronco cerebral. Una gran parte de los mensajes acústicos atraviesan la línea media del cerebro, alcanzando el tálamo por medio de los lemniscos laterales. Los estímulos auditivos al llegar a los cuerpos geniculados del tálamo, se proyectan a través de radiaciones auditivas, hasta alcanzar la circunvolución de Heschl del lóbulo temporal. En el área auditiva primaria, se registra las propiedades físicas de los sonidos, que serán procesados por la corteza auditiva (Portellano, 2007).

Por tanto, el uso de la tecnología ha permitido innovar en varios aspectos de manera positiva, así, una de las más destacadas es la educación, porque ha conseguido difundir libros, textos o aplicaciones a diferentes lugares del mundo, pero no ha conseguido llegar a todos los millones de niños debido al aspecto económico, por lo que, se produce una brecha tecnológica.

Así, vemos que en África 3 de cada 5 jóvenes que oscilan entre 15 y 24 años están fuera 
ISSN: 2600-5859

de línea, en cambio, en Europa la proporción es sólo 1 de cada 25. De este modo, en un mundo donde el 56\% de los sitios web están en idioma inglés, muchos niños no encuentran un contenido que puedan entender o que sea pertinente para sus vidas (UNICEF, 2017).

Después de todo, en Ecuador según el Instituto Nacional de Estadísticas y Censos INEC, indica que existe un incremento en 2017 del 12,1\% en el equipamiento de aparatos portátiles, tablet y laptop. Con respecto al 2012, 9 de cada 10 hogares del país tienen un teléfono inteligente.

Por lo cual, contar con tecnología en el hogar se ha constituido un servició básico. De igual forma, el acceso a internet se ha incrementado significativamente.

Por medio del Ministerio de Telecomunicaciones y de la Sociedad de la información (MINTEL), se generó una sociedad de internet que llega a localidades urbanas y rurales, permitiendo el acceso a las TIC en 6.700 instituciones educativas. Miles de docentes y estudiantes se favorecieron del equipamiento y de la conectividad.

Debido a que el uso de la tecnología es muy amplio en Ecuador, ha permitido que esta se aproveche en campos como la Estimulación Temprana. De acuerdo con estudios como la "Utilización de aplicaciones didácticas tecnológicas en el desarrollo del lenguaje de niños de 1-3 años que asisten al centro Baby Gym de la ciudad de Ambato", se evidencia que las aplicaciones didácticas tecnológicas inciden positivamente en el desarrollo del lenguaje de los niños (Jácome, 2015).

Además, el uso adecuado de la tecnología bajo la supervisión de un adulto puede facilitar el desarrollo integral de una persona. Debido a que los estímulos visuales a través de las TIC han proliferado no sólo en el intercambio de conocimientos, sino también en distribución de material sexual y otros de contenido ilegal que son utilizados para abusar y explotar a los niños.

En este sentido, de acuerdo con la UNICEF en 2017, el 92\% de todas las URL de abuso sexual infantil identificadas a escala mundial y de acuerdo con la Watch Foundation, están alojadas en solo cinco países, como Canadá, Estados Unidos, Rusia, Francia y los Países Bajos. Estos datos demuestran el mal manejo del uso de la tecnología en el mundo actual. Es por eso por lo que el empleo de la tecnología en edades tempranas debe ejecutarse bajo la supervisión de un adulto (Cánovas y Sahuquillo, 2013).

Actualmente, la tecnología se ha convertido en la base comunicativa de la humanidad, si recordamos el auge del iPad de Apple en el mercado en el 2010, dichos aparatos no llegaron solos, 
ISSN: 2600-5859

vinieron acompañados de diversas aplicaciones disponibles para cualquier tipo de aparato tecnológico. Además, estos se insertaron rápidamente en el ámbito infantil ya que se crearon aplicaciones didácticas que llamaba el interés del infante. Mientras que, que algunos padres de familia piensan que la tecnología sólo es un aporte positivo en su hogar, de entretenimiento, uso diario, ayuda a la realización de las tareas escolares, más bien se ha convertido en el aliado del aprendizaje y la adquisición de habilidades cognitivas, sociales, de comunicación y lenguaje.

Por otra parte, es un indicativo del porque la tecnología se ha insertado en las instituciones educativas cuyo fin es garantizar el aprendizaje interactivo y de fácil adquisición de conocimientos. Por ejemplo, las escuelas como Brains Nursery en Madrid. Aquí ya se habla de los beneficios de una educación tecnológica y con orientación a los padres. También se toma en cuenta la edad adecuada para el manejo de dispositivos y los tipos de contenidos. Asimismo, recordando que el acceso a estas tecnologías no debe ser de forma libre para los niños, sino con la supervisión de un adulto.

Según Espinar y López (2009) en su estudio afirman que cada vez el uso de la tecnología se produce en edades más tempranas. Por tanto, abundan las interrogantes sobre riesgos o beneficios que trae la tecnología, los avances que se ha conseguido gracias a esta que han marcado la historia de la humanidad. Entonces, si pensamos en la utilización de la tecnología como un exceso que trae consecuencias y un uso limitado, no llegaremos a aprovechar todos los beneficios que puede acarrear. Pero si la misma es correctamente empleada, los resultados serán favorecedores para quienes las manipule. Por ejemplo, tenemos el Primer Seminario Internacional de Tecnología y Educación en 2016, el cual hace énfasis a la tecnología como creadora de un ambiente activo, que permite tomar decisiones propias y mejorar la autoestima del estudiante (Pedro, 2016).

Por otra parte, el proyecto "Utilización de aplicaciones didácticas tecnológicas en el desarrollo del lenguaje de niños de 1-3 años que asisten al centro Baby Gym de la ciudad de Ambato", cuyo objetivo principal fue verificar los beneficios de las aplicaciones didácticas tecnológicas en el desarrollo del lenguaje en niños de 1 a 3 años. Vemos que los resultados mostraron que la utilización de aplicaciones didácticas tecnológicas permite que el niño adquiera un desarrollo de lenguaje acorde a su edad.

Es fundamental recordar que el desarrollo del lenguaje es un proceso complejo, el cual 
ISSN: 2600-5859

depende de los 3 primeros años de vida donde el cerebro se encuentra en su mayor plasticidad cerebral. Este periodo es intenso ya que la adquisición de las habilidades del habla y lenguaje requiere un ambiente que proporcione estímulos de sonidos e imágenes del habla de los padres.

Además, necesita la interacción con el mundo que lo rodea, es decir, con su familia. Por tanto, con estos datos investigados se consideró que si es posible realizar un estudio sobre la utilización de la tecnología en el desarrollo del lenguaje de niños que han cumplido 4 años.

Esta investigación busca describir el protagonismo que tiene la tecnología en los procesos de adquisición de las capacidades lingüísticas y también permite aclarar que aparte de gozar de una buena salud y aparatos fonológicos completos y bien desarrollados, existen factores externos que pueden interferir en el desarrollo del lenguaje.

EL proyecto se realizó en la Provincia de Tungurahua, Cantón Ambato, en la Unidad Educativa Sagrada Familia, durante el periodo de Marzo - agosto de 2019, de tal forma que se estima pueda contribuir y de alguna forma sea la base para próximas investigaciones. El uso de la tecnología es muy importe para el desarrollo de los pueblos, humanidad, pero al mismo tiempo, debe ser manejado con responsabilidad sobre todo en los primeros años de vida, donde el niño aprende de su entorno y será de vital importancia enviar estímulos de calidad siendo la tecnología un recurso favorable o al mismo tiempo pudiera ser desfavorable.

Fue factible ya que hubo la predisposición de los padres de familia y de los niños. Por lo tanto, dichas personas son beneficiarias directas de la investigación, puesto que los niños que son evaluados y se conoce de madera directa el nivel de lenguaje en el que se encuentran, así mismo, se puede emitir recomendaciones para mejorar o reforzar su desarrollo.

Los padres de familia no sólo adquieren pautas sobre el uso de la tecnología, si no también que se informan como emplear apropiadamente la tecnología en el hogar.

No obstante, los profesionales que laboran en el área de desarrollo infantil también son beneficiarios porque se observa que la tecnología no es negativa. Si se emplea de manera intencional y oportuna, de acuerdo con la edad y un horario establecido, se convierte en una herramienta idónea para la realización de las actividades con niños.

Este trabajo es de impacto para la comunidad, porque, se entiende que la tecnología no remplaza el juego real e imaginario. Por el contrario, es un aliado para el aprendizaje creando ambientes infantiles aptos para la utilización de la tecnología de forma positiva y adecuada. Por 
ISSN: 2600-5859

último, es necesario que existan más investigaciones sobre el empleo de la tecnología como una herramienta principal en ambientes de primera infancia, convirtiéndose así la escuela tradicional.

\section{Metodología}

Dentro del método es importante señalar que la investigación es comparativa, debido a que consiste en la generalización empírica y la verificación de hipótesis. A través de este se puede comprender cosas desconocidas a partir de las conocidas (Gómez y De la Garza, 2014). Esta investigación es de corte transversal, ya que se realizará una sola toma en un momento determinado a través del instrumento de evaluación.

\section{Participantes}

La población es finita ya que está claramente definida en tiempo y espacio (Paneque, 1998).

Se consideró como población a 34 niños de 4 años; 16 hombres y 18 mujeres que asisten al Inicial 2 de la Unidad Educativa Sagrada Familia.

Se conformó dos grupos de estudio; un normal y otro de control. Del grupo total de niños se dividió en partes iguales respectivamente.

Manteniendo los criterios de inclusión y exclusión tenemos: Ø Inclusión: Infantes de 4 años que asisten a la Unidad Educativa e infantes cuyos padres firman el consentimiento informado. Ø Exclusión: Infantes menores o mayores a 4 años de la Unidad Educativa e infantes que presenten alguna discapacidad; síndrome o trastorno.

\section{Diseño de Investigación}

En este estudio se ha utilizado un método cuali-cuantitativo, lo que permite analizarlos estadísticamente. Además, se empleó un diseño comparativo, con dos grupos de trabajo y con un corte transversal.

\section{Instrumentos y Técnicas de Recolección de Datos}

La técnica utilizada fue la observación. Los instrumentos de recolección de la información fueron un registro y el test de evaluación. En cuanto al test es importante mencionar que éste mide el desarrollo psicomotor TEPSI y ha sido creado para evaluar el desarrollo psicomotor de niños entre 2 y 5 años cumplidos. Además, es un tamizaje que determina si el desarrollo del niño es normal o bajo y de acuerdo con su edad. También, evalúa tres áreas principales, tales como: la coordinación, el lenguaje y la motricidad. Si el niño no alcanza el nivel que le corresponde, el test indica retraso (Haeussler y Marchant, 1999). Para el propósito de esta investigación se seleccionó 
ISSN: 2600-5859

el sub test lenguaje, que evalúa el lenguaje comprensivo y expresivo. Está constituido por 24 ítems a través de conductas, tales como: nombrar, describir, verbalizar situaciones u objetos, y describir escenas representadas en láminas (Haeussler y Marchant, 1999).

Se aplicó el sub test lenguaje del Test de desarrollo psicomotor TEPSI, cuyo objetivo fue medir el nivel de desarrollo del lenguaje de los niños y niñas de 4 años que asisten al Inicial 2 de la Unidad Educativa Sagrada Familia.

Plan de Análisis de Datos

Para la obtención de los datos se analizó las conductas expresadas en cada pregunta del test aplicado. Posterior se calculó el puntaje total del Sub test Lenguaje de los dos grupos de estudio.

Seguidamente, para verificar si existen diferencias y validar la hipótesis se aplicó el " $T$ Student" para 2 muestras relacionadas.

\section{Procedimiento}

El procedimiento de la investigación implicó tres fases:

La primera consistió en establecer el contacto y permiso de la institución para poder realizar la investigación. Además, la autorización del consentimiento informado para la participación de los niños.

La segunda fase fue la recolección de la información, permitiendo medir las variables propuestas a través del instrumento de evaluación en los dos grupos de estudio. En el grupo normal se utilizó láminas impresas de manera tradicional y en el grupo de control un dispositivo tecnológico, es decir, se adaptó el sub test Lenguaje empleando un computador. De esta forma se consiguió utilizar el test para cada participante.

La tercera fase se encargó del análisis de los datos obtenidos a través de la utilización del $P$ value. Con ello, se demostró la hipótesis planteada y se estableció las conclusiones y recomendaciones de los resultados obtenidos.

La investigación está basada en ciertos criterios éticos:

1) Solicitar permiso a las autoridades encargadas de la institución para el desarrollo de la investigación.

2) Para una mejor comprensión, facilitar información clara y precisa al participante acerca de la investigación planteada.

3) Utilización del consentimiento informado en el que se describe objetivos, procedimientos, 
ISSN: 2600-5859

aceptación y la interrupción de la participación del proyecto en cualquier momento.

4) Confidencialidad de los datos personales proporcionados en caso de publicar la investigación.

5) Velar por la seguridad del participante, con el fin de lograr beneficios y reducir riesgos.

El tipo de administración es de forma individual y el tiempo varía de acuerdo con la edad del niño, además, de la experiencia del examinador. Puede oscilar entre 30 a 40 minutos. La aplicación se realiza a niños que tengan la edad de 2 años, 0 meses, 0 días y niños de 5 años, 0 meses, 0 días.

Los criterios de evaluación se refieren a las conductas a valorar que están presentes en cada pregunta y existe dos formas de calificar; puede ser éxito o fracaso. Es decir, si la conducta resulta acertada se otorga un punto y si la conducta no es acertada, se otorga cero puntos. Además, cuenta con un manual de aplicación el cual explica con exactitud las conductas a observar para ser calificadas correctamente.

El supuesto de esta investigación fue: Hipótesis alterna (H1): La utilización de la tecnología influye en el desarrollo del lenguaje en niños de 4 años. Hipótesis nula (H0): La utilización de la tecnología no influye en el desarrollo del lenguaje en niños de 4 años.

\section{Resultados}

Los resultados mostraron que los puntajes del total PT del sub test Lenguaje en el grupo de control fueron más altos con relación al grupo normal. Véase figura 1. También se observó un mínimo de fracaso en el grupo de control por cada ítem evaluado. Véase figura 2.

A través del cálculo estadístico $p$ value, vemos que $\mathrm{P}=0.00000002846$ es menor al nivel de significancia $\sigma=0.05$. Por tanto, se rechazó la hipótesis nula (Ho) y se acepta la hipótesis alterna (H1), la cual se refiere a la utilización de la tecnología que si influye en el desarrollo del lenguaje en niños de 4 años. Véase figura 3. 
ISSN: 2600-5859

Vol. 3, $\mathrm{N}^{\circ} 1$, p. 63-79, marzo, 2020

\begin{tabular}{|c|c|}
\hline G. NORMAL & G. CONTROL \\
\hline Puntaje T & Puntaje T1 \\
\hline 52 & 61 \\
\hline 50 & 62 \\
\hline 43 & 60 \\
\hline 49 & 59 \\
\hline 53 & 60 \\
\hline 55 & 62 \\
\hline 43 & 61 \\
\hline 47 & 60 \\
\hline 53 & 59 \\
\hline 52 & 64 \\
\hline 53 & 60 \\
\hline 53 & 61 \\
\hline 50 & 60 \\
\hline 43 & 62 \\
\hline 47 & 59 \\
\hline 47 & 62 \\
\hline 56 & 59 \\
\hline
\end{tabular}

Figura 1

Puntajes totales del grupo normal y de control obtenidos del sub test lenguaje Fuente: Elaboración propia

\section{TEST NORMAL}

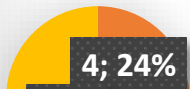

13;

$76 \%$

\section{TEST CON} TECNOLOGÍA

- Éxito 1

Fracaso 0
Éxito 1

Fracaso 0

Figura 2

Nombrar características de objetos

Fuente: Elaboración propia

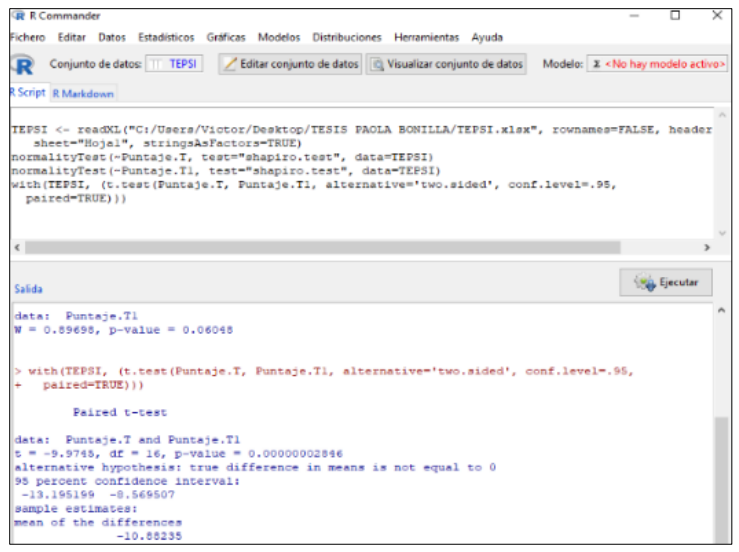

Figura 3

Cálculo del $p$ value

Fuente: Elaboración propia 
ISSN: 2600-5859

Vol. 3, N 1 , p. 63-79, marzo, 2020

En el grupo que se aplicó el sub test lenguaje de forma normal, se observa que existe 2 niños que corresponde al $12 \%$, se equivocan al contestar las preguntas, obteniendo un puntaje de 0 . Mientras que, el grupo de niños que usó tecnología vemos que el 100\% logró tener éxito consiguiendo un puntaje de 1 . A pesar de que existe un mínimo de fracaso en la respuesta de la pregunta planteada por el sub test con tecnología, es necesario hacer referencia como la tecnología favorece la capacidad individual del niño, generando autonomía y autocorrección. Puesto que las aplicaciones de internet son diseñadas para un fin, y si este no se consiguiese, existen pasos y opciones para desarrollar con éxito la actividad empleando varias repeticiones hasta que consiga dominar la misma. En el grupo que se aplicó el sub test lenguaje de forma normal, se observa que existe 2 niños que corresponde al 12\%, se equivocan al contestar las preguntas, obteniendo un puntaje de 0 . Mientras que, el grupo de niños que usó tecnología vemos que el 100\% logró tener éxito consiguiendo un puntaje de 1 . A pesar de que existe un mínimo de fracaso en la respuesta de la pregunta planteada por el sub test con tecnología, es necesario hacer referencia como la tecnología favorece la capacidad individual del niño, generando autonomía y autocorrección.

Puesto que las aplicaciones de internet son diseñadas para un fin, y si este no se consiguiese, existen pasos y opciones para desarrollar con éxito la actividad empleando varias repeticiones hasta que consiga dominar la misma. En la pregunta razona por analogías, se logró identificar que la tecnología al emplearse de manera positiva favorece el desarrollo cognitivo, sobre todo cuando existe un horario de acuerdo con la edad del niño y supervisión de un adulto.

De esta manera el contenido revisado en el dispositivo tecnológico permite mejorar el desarrollo del niño. De hecho, los grupos de estudio que se utilizaron en la aplicación del sub test lenguaje, adicionalmente, disponen de aparatos tecnológicos en el aula de clases, los cuales han favorecido el nivel de lenguaje de cada niño, como se refleja en los resultados. En el grupo de niños que se aplicó el sub test lenguaje de forma normal, se observa que 8 niños que corresponde al $47 \%$, no contestaron correctamente las preguntas y obtuvieron puntaje de 0 . Mientras que, el grupo de niños que usó tecnología vemos que el 100\% logro tener éxito y obtuvieron puntaje de 1. Estos resultados muestran que la descripción de escenas es más divertida frente a una pantalla, debido a que los niños mostraban mayor interés y mantenían la atención en cada imagen. También 
ISSN: 2600-5859

la claridad, nitidez y la gama de colores de las escenas aportaron en el éxito de la respuesta de las preguntas, sobre si describe o no escenas. En el grupo que se aplicó el sub-test lenguaje de forma normal, se observa que 6 niños que corresponde al 35\%, no respondieron correctamente las preguntas y obtuvieron puntaje de 0 . Al contrario, el grupo de niños que usó tecnología vemos que el 100\% logró tener éxito. El aporte positivo que permite observar el uso de la tecnología en el desarrollo del test, refleja la creación de un ambiente interactivo y motivador que permite al niño mantenerse despierto y sin aburrirse. Es por eso por lo que, a pesar del tiempo de duración de la evaluación, se sigue manteniendo el éxito en las respuestas para determinar el uso de plurales.

\section{Discusión}

El estudio demuestra claramente que la tecnología influye positivamente en el desarrollo del lenguaje en niños de 4 años. Al contrastar los resultados con el estudio realizado por Jácome (2015) en el centro Baby Gym de la ciudad de Ambato, los resultados mostraron que las aplicaciones didácticas tecnológicas permiten que el niño adquiera un desarrollo del lenguaje acorde a su edad. Vemos que, efectivamente, la tecnología es una herramienta de ayuda para el desarrollo del lenguaje, debido a que los niños se comportaban más activos y motivados. A pesar del tiempo de exposición a estas actividades no se sentían cansados o aburridos.

En un estudio realizado por Remache (2015), los resultados obtenidos de la aplicación del programa Spanish Artic aplicado a niños con dislalia funcional de 4 a 6 años, no proporcionó los resultados esperados a causa de la excesiva cantidad de actividades disponibles en dicho programa.

Puesto que, para integrar la tecnología en procesos de desarrollo infantil es necesario que el profesional se encuentre capacitado y preparado pedagógicamente acerca del empleo de la tecnología. De manera simultánea en el trabajo con los niños, como lo demostró Jin (2016) en su estudio de posgrado, sugiere que el aprendizaje mediado por las nuevas tecnologías se deriva del conocimiento generado por el maestro sobre el uso de la tecnología en lugar de la tecnología.

Cabe considerar, que las nuevas tecnologías son influyentes en el desarrollo del adolescente y se explica esto ya que un alto porcentaje de usuarios usa TIC para fines de comunicación y entretenimiento.

Evidentemente estos resultados se relacionan con los cambios en las costumbres, hábitos y actitudes de las generaciones actuales, concluyen Barquero y Calderón (2016), en su análisis dirigido a una población mayormente expuesta a las tecnologías. Al mismo tiempo, el aprendizaje 
ISSN: 2600-5859

vicario sobresalta en los hogares actuales, debido a las conductas de imitación ejercida por un niño o niña a los miembros del hogar. Por esta razón el uso de la tecnología es cada vez más temprano (Villadangos, 2009).

Sin duda alguna, es fundamental formar una sociedad que empleé la tecnología de una manera segura, puesto que existe cada vez personas más jóvenes manipulando y experimentando en ella.

\section{Conclusiones}

Es esencial destacar que, en la investigación realizada se aplicó nuevas tecnologías considerando los hitos de desarrollo de acuerdo con la edad de la población expuesta. Del mismo modo, se consideró el tiempo de uso de la tecnología recomendado a los niños de 4 años.

En el análisis procedente de los datos, vemos que el grupo de control mostró mayor puntaje al contrario del grupo normal; a pesar de que toda la población en su instrucción escolar cuenta con la interacción de aparatos tecnológicos. En definitiva, el empleo de la tecnología motivó a un buen ambiente de trabajo para el desarrollo de cada actividad del sub test. De esta forma, se concluye que la tecnología puede ser una buena práctica si se utiliza el instrumento adecuado y con supervisión de un profesional que esté capacitado e informado acerca de las nuevas tecnologías de la información y comunicación.

Es importante mencionar las limitaciones que se presentaron durante la investigación, las cuales se despliegan sobre la desinformación de la integración de la tecnología en campos de desarrollo del niño de forma planificada y estructurada.

La investigación determinó la influencia de la tecnología en el desarrollo del lenguaje de niños y niñas de 4 años como una herramienta de ayuda para el proceso de aprendizaje, que prioriza recursos, mejora la autonomía, favorece la comprensión y está ligada a la actividad de la sociedad actual.

La aplicación del sub test de lenguaje de la prueba de desarrollo psicomotor TEPSI, arrojo como resultado, tanto para el grupo normal, así como para el grupo de control, un nivel de desarrollo adecuado para la edad del niño. Sin embargo, las conductas presentadas en los niños del grupo de control fueron más favorables, puesto que los niños mostraron mayor actividad, concentración y motivación. A pesar del tiempo de evaluación no perdieron la atención y tampoco se mostraban cansados. Al contrario, en el grupo normal se mostraron distraídos y algo fatigados. 
ISSN: 2600-5859

Como consecuencia vemos que la tecnología permite mantener a los niños activos y motivados, además hay que tener en cuenta que son nativos digitales.

Se recomienda emplear el uso de la tecnología a personas con discapacidad, debido a la creación de varias aplicaciones y de acuerdo con las necesidades de las personas, que favorecen la autonomía, el aprendizaje, la autoconfianza y, con ello, potenciar sus habilidades. Asimismo, crear ambientes estimulantes en el hogar desde el primer año de vida para favorecer el desarrollo integral del niño, así como el área de lenguaje a través de sonidos, canciones, conversaciones o la utilización de medios tecnológicos, pero siempre con la orientación permanente de un adulto.

En cuanto a líneas de investigación futuras, hay que señalar que existe un amplio horizonte de posibilidades como, por ejemplo, las aportaciones de la tecnología en una terapia infantil o estructuración de instrumentos de valoración para el desarrollo integral del niño. Todo esto a través de medios digitales, así como, beneficios y efectos negativos de terapias asistidas tecnológicamente.

\section{Referencias bibliográficas}

Alfonso, C., \& López, P. I. (2013). Estimulación del desarrollo en la infancia: inteligencia, lenguaje, afectividad y otras áreas. Dykinson.

Baquero, A., \& Calderón, F. (2016). Influencia de las Nuevas Tecnologías en el Desarrollo Adolescente y posibles desajustes. Revista Cúpula, 30(2), 11-25. Retrieved from http://www.binasss.sa.cr/bibliotecas/bhp/cupula/v30n2/art02.pdf.

Cabero, J., Córdoba, M., \& Fernández, J. M. (2010). Las TIC para la igualdad. Educación Siglo XXI, 28(1), 353-356.

Cánovas Leonhardt, P., \& Sahuquillo Mateo, P. M. (2013). La protección del menor en las familias. Edetania, (44), 93-109.

Cordoba, M., Fernández, J. M., \& Cabero, J. (2012). Las TIC para la igualdad. Nuevas tecnologías y atención a la diversidad: Aula múltiple Magisterio.

Childe, G. (1996). Los orígenes de la civilización. Fondo de Cultura Económica.

Espinar Ruiz, E., \& López Fernández, C. (2009). Jóvenes y adolescentes ante las nuevas tecnologías: percepción de riesgos. Athenea digital: revista de pensamiento e investigación social, (16), 001-020. 
ISSN: 2600-5859

Gómez Díaz de León, C., \& de la Garza, L. (2014). Método comparativo. http://eprints.uanl.mx/9802/1/Estudio\%20Comparado.pdf.

Haeussler, I. M., \& Marchant, T. (1999). TEPSI: Test de desarrollo psicomotor 2-5 años. Universidad Católica de Chile.

Jácome Jácome, J. G. (2015). Utilización de aplicaciones didácticas tecnológicas en el desarrollo del lenguaje de niños de 1-3 años que asisten al Centro Baby Gym de la ciudad de Ambato (Bachelor's thesis, Universidad Técnica de Ambato-Facultad de Ciencias de la SaludCarrera de Estimulación Temprana).

Jin, M. (2016). Technology integration in the context of collaboration in early childhood settings (Order No. 10297017). Available from ProQuest Central. (1847569269). Retrieved from https://search.proquest.com/docview/1847569269?accountid=36765.

Paneque, R. J. (1998). Metodología de la investigación: elementos básicos para la investigación clínica. Editorial Ciencias Médicas. Recuperado de: http://www.sld.cu/galerias/pdf/sitios/rehabilitacion-doc/metodologia_dela_investigaciontexto.pdf.

Pedro, F. (2016). Tecnologías para la transformación de la educación. Madrid: Fundación Santillana.

Portellano, J. A. (2007). Neuropsicología infantil (No. 616.8: 159.9-053.2). Síntesis.

Quintanilla, M. Á. (1998). Técnica y cultura. Teorema: Revista Internacional de Filosofía, 49-69.

Remache Guastay, R. J. (2015). Aplicación del programa Spanish artic en el desarrollo del lenguaje de los niños con dislalia funcional de 4 a 6 años que acu-den al centro de estimulación temprana Waikiki (Bachelor's thesis, Universidad Técnica de Ambato-Facultad de Ciencias de la Salud-Carrera de Estimulación Temprana).

UNICEF. (2017). Estado Mundial de la Infancia 2017: niños en un mundo digital. Resumen.

Villadangos, S. M., \& Labrador, F. J. (2009). Menores y nuevas tecnologías (NT): ¿uso o abuso. Anuario de Psicología Clínica y de la Salud, 5(1), 75-83.

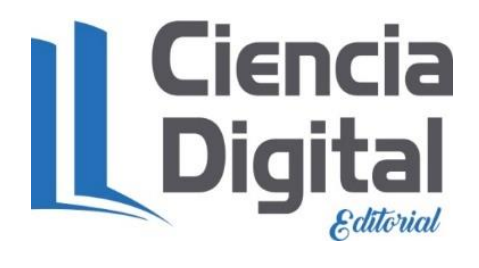


ISSN: 2600-5859

\section{PARA CITAR EL ARTÍCULO INDEXADO}

Bonilla Morales, P. de los Ángeles, Troya Ortiz, E. V., \& Peñafiel Gaibor, V. (2020). La utilización de la tecnología en el desarrollo del lenguaje de los niños de 4 años. ConcienciaDigital, 3(1.2), 6379. https://doi.org/10.33262/concienciadigital.v3i1.2.1174

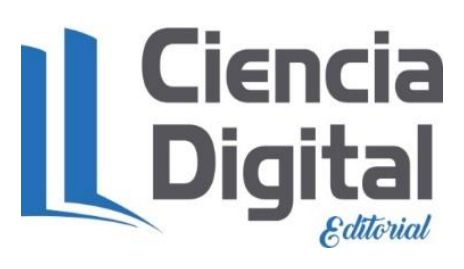

El artículo que se publica es de exclusiva responsabilidad de los autores y no necesariamente reflejan el pensamiento de la Revista Conciencia Digital.

El artículo queda en propiedad de la revista y, por tanto, su publicación parcial y/o total en otro medio tiene que ser autorizado por el director de la Revista Conciencia Digital.

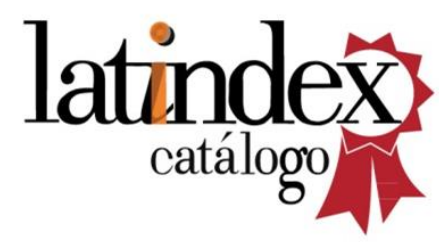

Conciencia

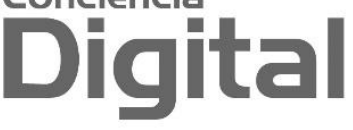

